\title{
Gentilitas y paganismus en el sermón XLVIII de Máximo de Turín
}

Recibido: 27/07/2018. Aceptado: 07/05/2019

\section{Resumen}

En base al análisis de las líneas 78-102 del sermón 48 de Máximo de Turín, este trabajo tiene un doble objetivo: por un lado, examinar la pertinencia de la acríticamente aceptada sinonimia que, de acuerdo a los investigadores, habría existido en la prédica del obispo entre los términos paganismus y gentilitas; por otro, determinar la especificidad semántica que a cada uno de ellos correspondió a partir de la consideración de las características que el autor atribuyó respectivamente a pagani y gentiles en dicha homilía.

PALABRAS CLAVE: Máximo de Turín; Sermón 48; Paganismo; Gentilidad; Sinonimia.

Gentilitas and Paganismus in the Sermon 48 of Maximus of Turin

\begin{abstract}
Based on the analysis of Maximus of Turin's sermon 48.78-102, this paper pursues a double objective: on the one hand, to examine the relevance of the uncritically accepted synonymy that, according to the researchers, exists in his Sermones between the terms paganismus and gentilitas; on the other hand, to consider the characteristics Maximus respectively assigned to pagani and gentiles in order to establish the semantic specificity that corresponded to each term in this sermon.
\end{abstract}

KEYWORDS: Maximus of Turin; Sermon 48; Paganism; Gentilitas; Synonymy. 


\section{Introducción}

Ninguno de los estudiosos que en el curso de la última centuria se han aproximado a la consideración del discurso que Máximo de Turín (...398...423) elaboró en sus Sermones ${ }^{1}$ a propósito del 'paganismo', ${ }^{2}$ ha creído necesario presentar una definición del fenómeno analizado. El carácter inespecífico del objeto de estudio no ha impedido, sin embargo, la formulación de una serie de afirmaciones que, con el paso de los años, se han constituido en verdaderos axiomas: en primer lugar, la certeza de que el 'paganismo' - fuese lo que fuese - gozó de una sólida presencia en Turín y sus alrededores durante el episcopado maximiano; ${ }^{3}$ luego, la convicción de que el error ${ }^{4}$ la superstitio, ${ }^{5}$ los idola,${ }^{6}$ los dii, ${ }^{7}$ los daemones, ${ }^{8}$ las arae, ${ }^{9}$ los pecudum capita adfixa liminibus, ${ }^{10}$ las festiuitates, ${ }^{11}$ las superstitiones, ${ }^{12}$ los omina ${ }^{13}$, la immolatio $^{14}$ - entre muchas otras entidades y prácticas que el obispo localizaba, principal pero no exclusivamente, en la campaña turinesa-, constituían manifestaciones de tal presencia; finalmente, la creencia de que esta "Gefahr [...] für den Christen", ${ }^{15}$ esta "piaga profondamente radicata e diffusa", ${ }^{16}$ "preoccupava non poco il nostro vescovo", ${ }^{17}$ lo "obsesionaba", ${ }^{18}$ era advertida "come grave" ${ }^{19}$ por el obispo, quien, en consecuencia, habría

\footnotetext{
1 En todos los casos seguiremos la edición de Mutzenbecher (1962). En las notas al pie referiremos a los sermones como 'S.'. Todas las traducciones son propias.

2 El presente trabajo se basa en la certeza de que Máximo empleó el término paganismus en un sentido muy diferente respecto de aquel en el cual los estudiosos modernos lo han comprendido. En consecuencia, y a fin de diferenciar claramente las ocasiones en que aludimos al paganismus maximiano de aquellas en que referimos al 'paganismo' de los estudiosos de su vida y de su obra, emplearemos el término latino en cursiva para lo primero, en tanto que el término castellano y comillas para lo segundo.

3 Resulta imposible referir aquí a la totalidad de la bibliografía relativa a la vida y a la obra de Máximo de Turín en la cual se advierte la asociación sea de uno u otro de estos fenómenos o de su conjunto con el 'paganismo'. Ante ello, remitimos únicamente a los estudios que, sea en función de su relevancia en el marco de la bibliografía maximiana o de su actualidad, resultan más significativos: Gallesio (1975:26-27), Devoti (1981:156,162,165), Spinelli (1983:39), Ramsey (1989:7), Lizzi (1989:197), Banterle (1991:15), Zangara (1992:517), De Filippis Cappai (1995:53-54), Plazanet-Siarri (1996:9-10,14); Merkt (1997:106-108), Bolgiani (1998:123), De Filippis Cappai (1999:53), Devoti (1999:101-102,115), Maritano (1995:129-130), Bolgiani (1997:268-269), Mariani Puerari (1999:10, 64, 83), Banterle (2003:17), Eger (2014:46), Ariano M.L. (2014:147155), Ariano M.P.A. (2014:234-240), Ariano M.L. (2017:29-30). Para un análisis en profundidad de la historiografía maximiana, véase Noce (2017:177-202).
}

4 S. 30. 52-53; S. 31.6; S. 48.80; S. 61c extr.65-66; S.98 extr.4; S. 105 extr.35-36; S. 106 extr.24-25; S. 107 extr.46.

5 S. $1.36 ;$ S.61c extr.72; S.63.5; S. 73.100; S.98 extr.4-6,17-19; S. 101 extr.7-11; S. 103 extr.28; S. 106 extr.5.

6 S. 63.12; S. 91 extr.23-24; S. 105 extr.40-43; S. 106 extr.45-47; S. 107 extr.4-6,22.

7 S. 91 extr.9; S. 107 extr.32-35,38-44.

8 S. 91 extr.25-26; S. 101 extr.7-11; S. 107 extr.31-32.

9 S. 91 extr.27-28; S. 107 extr.32-35.

10 S. 91 extr.28.

11 S. 61c extr.70-71; S. 63.4,35-36; S. 98 extr.17-19; S. 101 extr.7-11.

12 S. 1.36; S. 61c extr.72; S. 63.5; S. 73.100; S. 98 extr.4-6, 17-19; S. 101 extr.7-11; S. 103 extr.28; S. 106 extr.5. 13 S. 61c extr.70-71; S. 63.4,35-36; S. 98 extr.17-19; S. 101 extr.7-11.

14 S. 91 extr.30-31; S. 107 extr.12-16,24-25.

15 Merkt (1997:105).

16 Spinelli (1983:39).

17 Ariano M.P.A. (2014:219, en igual sentido 224).

18 Bolgiani (1997:269).

19 Ariano M.L. (2017:29-30). 
tenido entre sus principales preocupaciones ${ }^{20}$ "la lotta senza quartiere alle sopravvivenze dei culti pagani e a tutto ciò che sapeva di paganesimo", ${ }^{21}$ combate afrontado con "passione", ${ }^{22}$ con un cierto "aria di crociata" ${ }^{23}$

A nuestro entender, se debe conceder a la historiografía maximiana un acierto: Máximo, efectivamente, consideraba el paganismus un fenómeno negativo. Pero en este punto finalizan las similitudes existentes entre el paganismus maximiano y el 'paganismo' de la historiografía: por una parte, Máximo hizo referencia al paganismus en apenas una de las 111 homilías consideradas auténticas o dudosas por su editora, Almut Mutzenbecher. Demasiado poco para aceptar que, a decir de Máximo, constituía una problemática digna de destacada atención; por otra, las entidades y prácticas antes mencionadas fueron en todos los casos caracterizadas por el autor como manifestaciones no de paganismus sino de gentilitas.

Ante estas circunstancias, nos hemos preguntado si la pretendidamente mayúscula preocupación del obispo por el 'paganismo' no sería acaso consecuencia menos de una realidad histórica que de un error historiográfico. Dilucidar tal circunstancia, comprender la naturaleza del presunto error $y$, una vez hecho esto, procurar superarlo son los objetivos de este trabajo.

\section{Gentilitas y paganismus en la bibliografía maximiana}

El origen de la confusión se identifica claramente si consideramos el empleo que de ciertos términos realizaron los traductores de los Sermones maximianos a distintas lenguas modernas así como los comentaristas de la vida y obra del obispo de Turín. Unos pocos ejemplos serán suficientes. Comencemos observando una tabla que nos permite apreciar la discordancia existente entre el empleo efectivo del término paganus en los Sermones de Máximo y las traducciones hoy en día existentes:

\begin{tabular}{|c|c|c|c|c|c|c|c|}
\hline Editor & $\begin{array}{c}\text { Edición latina } \\
\text { (Mutzenbecher) }\end{array}$ & Gallesio $^{24}$ & Padovese $^{25}$ & Ramsey $^{26}$ & Banterle $^{27}$ & $\begin{array}{c}\text { Plazanet- } \\
\text { Siarri }^{28}\end{array}$ & $\begin{array}{c}\text { Mariani } \\
\text { Puerari }^{29}\end{array}$ \\
\hline $\begin{array}{c}\text { Sermones auténticos y dudosos } \\
\text { reunidos en la edición }\end{array}$ & 111 & 67 & 42 & 111 & 111 & 42 & 55 \\
\hline $\begin{array}{c}\text { Cantidad de veces que se utilizan } \\
\text { los términos paganus o paganismus }\end{array}$ & 2 & 7 & 12 & 16 & 28 & 13 & 17 \\
\hline $\begin{array}{c}\text { Cantidad de sermones en que se } \\
\text { utilizan tales términos }\end{array}$ & 1 & 5 & 7 & 9 & 21 & 11 & 11 \\
\hline $\begin{array}{c}\text { Porcentaje de los sermones en que } \\
\text { se utilizan tales términos }\end{array}$ & $0,9 \%$ & $7,46 \%$ & $16,66 \%$ & $8,10 \%$ & $18,91 \%$ & $26,19 \%$ & $20 \%$ \\
\hline
\end{tabular}

20 Merkt (1997:75), Di Mauro (2001:71), Eger (2014:4-5), Ariano M.L. (2014:147-148).

21 Bolgiani (1997:268). En similar sentido Devoti (1981:156,165-166).

22 Ariano M.P.A. (2014:216).

23 De Filippis Cappai (1995:54).

24 Gallesio (1975).

25 Padovese (1985).

26 Ramsey (1989).

27 Banterle (2003).

28 Plazanet-Siarri (1996).

29 Mariani Puerari (1999). 
Como se ve, no todo aquello que los traductores trasladaron por 'pagano' y 'paganismo' da cuenta de lo que Máximo denominaba paganismus. En otras palabras, los traductores se han mostrado muy proclives a encontrar testimonios de 'paganismo' donde el obispo no parece haber dado cuenta de tal fenómeno.

Una segunda tabla, en tanto, evidencia que en una muy buena cantidad de casos el 'paganismo' de los traductores procura dar cuenta de los vocablos gentilis o gentilitas, los cuales emergen un total de 29 veces a lo largo de las homilías maximianas:

\begin{tabular}{|c|c|c|c|c|c|c|c|c|}
\hline & \multirow[t]{2}{*}{ Ubicación } & \multirow{2}{*}{$\begin{array}{c}\text { Término } \\
\text { latino }\end{array}$} & \multicolumn{6}{|c|}{ Traducción } \\
\hline & & & Gallesio & Padovese & Ramsey & Banterle & $\begin{array}{l}\text { Plazanet- } \\
\text { Siarri }\end{array}$ & $\begin{array}{l}\text { Milena } \\
\text { Puerari }\end{array}$ \\
\hline 1 & I, 37 & Gentilium & - & - & peoples & Pagani & Païens & - \\
\hline 2 & II, 55 & Gentilium & pagani & ---- & nations & Gentili & Païennes & --- \\
\hline 3 & $X X, 55$ & Gentilitatis & --- & - & paganism & paganesimo & --- & --- \\
\hline 4 & $\mathrm{XXXI}, 6$ & Gentili & - & - & pagan & Pagano & - & - \\
\hline 5 & XXXIII, 83-84 & Gentilitatis & paganesimo & --- & nations & paganesimo & --- & - \\
\hline 6 & $X X X V, 87$ & Gentilium & --- & pagani & Gentiles & Pagani & Païen & Pagani \\
\hline 7 & XLVIII, 79 & gentilium & & ---- & Gentiles & gentili & ---- & - \\
\hline 8 & XLVIII, 83 & gentilium & ---- & ---- & Gentiles & gentili & ---- & ---- \\
\hline 9 & XLVIII, 97 & gentilis & & ---- & pagan & gentile & ---- & ----- \\
\hline 10 & XLVIII, 99 & gentilis & & --- & Gentile & pagano & ---- & - \\
\hline $\begin{array}{l}11 \\
12\end{array}$ & $\begin{array}{l}\text { LXIc extr., } 67 \\
\text { LXIc extr., } 70\end{array}$ & $\begin{array}{l}\text { gentilitatis } \\
\text { gentilibus }\end{array}$ & - & $\begin{array}{l}\text { paganesimo } \\
\text { pagani }\end{array}$ & $\begin{array}{l}\text { pagan } \\
\text { pagans }\end{array}$ & $\begin{array}{l}\text { pagane } \\
\text { pagani }\end{array}$ & --- & $\begin{array}{l}\text { pagane } \\
\text { pagani }\end{array}$ \\
\hline 13 & LXII, 4 & gentiles & ---- & pagani & pagans & Gentili & Gentils & Pagani \\
\hline 14 & LXIII, 4 & gentilium & --- & pagani & pagan & pagani & ---- & pagani \\
\hline 15 & LXIIII, 35 & gentilium & --- & pagani & pagans & pagani & ---- & pagani \\
\hline 16 & LXIII, 39 & gentilium & ---- & pagani & pagans & gentili & ---- & pagani \\
\hline 17 & LXXIII, 47 & gentilis & pagano & ---- & pagan & pagano & ---- & ---- \\
\hline 18 & LXXIII, 96 & gentilium & ---- & ---- & pagans & pagani & ---- & ---- \\
\hline 19 & LXXXIV, 89 & gentilium & --- & ---- & Gentile & Pagani & --- & pagani \\
\hline 20 & LXXXV, 58-59 & gentilis & $-{ }_{-1}$ & 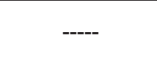 & sacrilegious & Pagano & --- & -.- \\
\hline 21 & LXXXVIII, 54 & gentilis & ---- & ----- & Gentile & Pagano & Gentils & ---- \\
\hline 22 & XCl extr., 28 & gentilium & pagane & - & pagans & pagani & --- & --- \\
\hline 23 & XCl extr., 38 & gentiles & pagani & ---- & pagans & pagani & ---- & --- \\
\hline 24 & XCVIII extr., 3 & gentilium & ---- & pagane & Gentiles & pagani & païennes & pagani \\
\hline 25 & XCVIII extr., 12 & gentilium & & pagani & Gentiles & pagani & idolâtrie & pagani \\
\hline 26 & $\mathrm{Cl}$ extr. 8 & gentiles & ---- & pagani & Gentiles & Gentili & Païens & pagani \\
\hline 27 & CIII extr., 28 & gentilium & $-{ }_{-1}$ & pagani & Gentiles & Pagani & Païenne & pagani \\
\hline 28 & CVI extr., 13 & gentiles & --- & ---- & Gentiles & Pagani & --- & ---- \\
\hline 29 & CVII extr., 6 & gentilitatis & ---- & ---- & Gentiles & paganesimo & ---- & --- \\
\hline
\end{tabular}


De este modo, dos circunstancias resultan claras: por un lado, que la especificidad semántica de los términos gentilis y gentilitas no preocupó en nada a los traductores. Por el contrario, concedieron a estos vocablos una flexibilidad tal que derivó en traslaciones múltiples: 'gentil', 'pagano', 'nación', 'pueblo', 'gens' e incluso 'sacrilegio'; por otro, que la destacada presencia cuantitativa que el 'paganismo' adquiere en dichas traducciones y - como veremos - en los estudios de los comentaristas es consecuencia de la asunción de que allí donde el texto latino empleaba gentilis o gentilitas resultaba correcto suponer la presencia de 'paganos' o 'paganismo'.

Similar modo de proceder se aprecia también en los estudios sobre la vida y la obra maximiana. Veamos unos ejemplos. En sintonía con los lineamientos historiográficos predominantes, Lino Spinelli aseveró que "L'azione pastorale di san Massimo trovò grossi ostacoli soprattutto nei pagani, negli eretici e nei giudei" (1983:39). Respecto de los primeros añadía:

Il paganesimo nell'ambiente piemontese sembra una piaga profondamente radicata e diffusa. Gli accenni ad idoli e divinità, feste e culti di superstizione praticati talvolta anche dai cristiani, sono continui. San Massimo ne tratta esplicitamente in vari sermoni. (1983:39)

Tal aseveración, indicaba el autor en nota al pie, se sustentaba en los sermones 63, 91 extr., 98 extr., 106 extr., 107 extr. y 198 extr..$^{30}$ Ahora bien, en ninguna de tales homilías se aprecian los términos paganus ni paganismus. Por el contrario, excepción hecha del sermón 108 extr., todas ellas emplean los vocablos gentilis o gentilitas. La aceptación de una sinonimia entre ambos resulta, pues, evidente.

Idéntico es el caso de Franco Bolgiani. El autor afirmó que "la lotta senza quartiere alle sopravvivenze dei culti pagani e a tutto ciò che sapeva di paganesimo" (1997:268) había constituido una de las más importantes tareas pastorales emprendidas por Máximo. Vale la pena citar el extenso pasaje de su trabajo en el cual reunía los argumentos que, a su entender, refrendaban tal aseveración:

Sono i proprietari terrieri abitanti in città i primi a essere richiamati dal vescovo: per non urtarsi con i servi o villici alle loro dipendenze, fingono di non vedere, tollerano, sono reticenti, non si impongono, mentre dovrebbero procedere, impedire, reprimere, cancellare e far sparire ogni traccia di «gentilitas». L'idolatria, dichiara Massimo, è "grande malum», è un «inquinamentum», una «pollutio» che contamina chi abita sul territorio dove rimangono idoli: infetta chi anche solo li guarda, attacca anche i ministri di Dio, raggiunge coloro che sono consapevoli così come coloro che tacciono. Basta che un «rusticus» faccia un sacrificio (pagano) che il padrone, a distanza, ne è contaminato, poiché chi non impedisce è come se facesse. La presenza di «demonismo pagano» nelle campagne sembra ossessionare il vescovo che ne teme la tenace presa sugli abitanti del luogo. Egli va in giro fuori della città e scopre che non c'è quasi proprietà immune dal culto dei demoni: altari, tempietti, simulacri di pietra, crani di animali esposti sulla soglia delle case, tracce ancor calde di sacrifici recenti, «omnia ibi inquinata, omnia sunt nefanda». Massimo invita a dar la caccia ai superstiti sacerdoti di questi modesti riti agrari a cui riserva sarcastico disprezzo: pazzi, ubriaconi, «col capo irsuto di scomposte chiome posticce», il petto nudo, «il mantelletto sino a metà della gamba», che con una lama in mano si infliggono ferite, quasi gladiatori autoaffligentisi. Tratti 
di osservazione realistica e probabili echi letterari di culti orgiastici di origine orientale entrano, come si vede, nella descrizione di Massimo.(Bolgiani, 1997:268-269)

De acuerdo a Bolgiani (1997:268-269, notas 172-177), sus consideraciones se sustentaban en los sermones 17, 91 extr., 102 extr., 106 extr., 107 extr. y 108 extr. Otra vez, en tanto que ninguno de ellos contiene el término paganus, las homilías 91 extr. y 106 extr. aluden a los gentiles.

Resulta evidente, pues, que tanto los traductores de los Sermones a lenguas modernas como los estudiosos de la vida y de la obra del obispo de Turín han asumido que: 1) el obispo empleaba los términos gentilis-gentilitas y paganuspaganismus como sinónimos para referir a un mismo colectivo histórico; 2) que ese colectivo histórico se corresponde con lo que ellos mismos han denominado 'paganismo'; 3) en consecuencia, que la gentilitas-paganismus maximiano constituía, a decir del obispo, un elemento social negativo, peligroso para el cristianismo y, por ello, objetivo de una lucha encarnizada.

Claro está, esta secuencia de afirmaciones $-\mathrm{y}$, con ello, la verosimilitud de la interpretación tradicional y hegemónica respecto del discurso maximiano respecto del 'paganismo' - depende, en última instancia, de la veracidad de la primera de ellas. Si gentilitas y paganismus no constituyeron sinónimos en la prédica del obispo de Turín, entonces no se sostendrían ni, por un lado, la correspondencia entre la gentilitas-paganismus maximiana y el 'paganismo' de los investigadores ni, por otro, la caracterización que respecto del fenómeno se ha realizado. La cuestión clave, pues, es dilucidar a partir de su propia prédica si gentilitas y paganismus constituyeron o no sinónimos para Máximo. A tal fin, resulta fundamental considerar, siquiera brevemente, el sermón 48 , puesto que no solo contiene las dos únicas utilizaciones del término paganus presentes en lo que de la homilética maximiana ha llegado hasta nosotros, sino que, además, emplea también el vocablo gentilis en cuatro ocasiones. Esta circunstancia permite comparar las características que el obispo asignó respectivamente a los pagani y a los gentiles y, con ello, evaluar la pretendida sinonimia.

\section{El sermón 48}

Nos limitaremos aquí a la consideración de las líneas 78 a 102, ${ }^{31}$ aquellas en las cuales los antedichos términos se hacen presentes. En ellas, Máximo trataba sobre Mateo 18, 6: Jesús advertía en dicho versículo que, a quien escandalizase a uno de los pequeños que creían en él, más le valdría ser arrojado a lo profundo del mar con una piedra de molino de asno atada al cuello. Veamos el texto:

Namque in scripturis in asini figura populus gentilium nuncupatur, qui clausis oculis circa ignorantiae molam erroris sui gyro pertrahitur, et sine ulla cursus directione uestigiis in se saepe redeuntibus inuitus usui laborat alieno. Nonne enim tibi uidetur molam asinariam uoluere gentilium populus, quamdiu in inperitiae suae labore uersatur? Naturae quidem uinculis alligatus, ut uerbum

31 Aunque indudablemente contribuiría a la contextualización del pasaje que aquí analizaremos, por diversos motivos consideramos inoportuno presentar el comentario de la totalidad del sermón XLVIII en esta ocasión: por un lado, porque extendería el presente trabajo más allá de lo adecuado; por otro, porque supondría una modificación radical de su naturaleza originaria, a saber y tal como se ha dicho, la de una exposición en el marco de un evento científico; finalmente, porque hemos realizado ya un análisis de tal tipo en Noce (2019: 187, 190-195), artículo al que remitimos. 


\begin{abstract}
dei molat dominum caeli quaerat; sed opertae mentis caecitate suffusus, ut uultum animi erigere ad deum cordis oculos leuare non possit ad caelum. Sed quoniam molam asinariam saxum esse constat, uideamus si et paganorum talis est mola! Talis plane est; saxea enim mola est paganorum Iuppiter Herculesque lapideus, circa quos caecis oculis frequenti errore circueunt. Caecos, inquam, dixerim eorum oculos qui putant se uidere quod non uident. Aspicientes enim saxum deum se uidere confidunt. At tamen qui molam uoluit consummandi aliquando fidem operis habet, exuendae spem gerit necessitatis; cui uero mola asinaria ad collum suspenditur portat lapidem, qui portare iugum domini recusauit. Asinus ergo ad molam caecus ad lapidem gentilis ad saxum, qui adorat eum quem non uidet nec agnoscit. Deus enim non in manufactis habitat, nec in metallo aut saxo cognoscitur. Hic ergo gentilis populus, cum persecutionem christiano intulerit, hac poena plectetur, ut cum sua sacrilega mola iudicandi saeculi fluctibus demergatur ${ }^{32}$
\end{abstract}

Ciertamente, con la figura del asno se representa en las Escrituras al pueblo de los gentiles, el cual, con los ojos cerrados, es llevado por la fuerza en el círculo de su error alrededor de la muela de la ignorancia, y sin ninguna dirección en su andar, pasando las huellas de sus pies muchas veces sobre sí mismos, involuntariamente se fatiga en beneficio ajeno. ¿No te parece, en efecto, que el pueblo de los gentiles gira una muela de molino de asno mientras se halla envuelto en los pesares de su impericia? En verdad está obligado por las ataduras de la naturaleza a moler la palabra de Dios y a buscar al Señor del cielo, pero inundado por la ceguera de su mente abrumada, no puede levantar la mirada de su alma hacia Dios ni alzar los ojos del corazón hacia el cielo. Pero puesto que consta que la muela de molino de asno es una piedra, veamos si también es tal la muela de molino de los paganos. Ciertamente es tal: en efecto, muela de molino de piedra de los paganos es Júpiter y Hércules lapídeo, en torno a los cuales giran con ojos ciegos con repetido error. Ciegos, digo, llamaría a sus ojos, que creen ver lo que no ven. Mirando una piedra, confían en que ven un dios. No obstante, quien gira la muela de molino tiene fe en que alguna vez su tarea vaya a terminar, tiene la esperanza de que la constricción va a ser apartada de sí. Pero una muela de molino de asno se le cuelga al cuello a quien acepta una piedra, quien rechazó aceptar el yugo del Señor. Quien adora lo que no ve ni conoce es, pues, un asno en la muela, un ciego ante la piedra, un gentil en la piedra. Dios, en efecto, no habita en las obras de los hombres, ni se lo reconoce en el metal ni en la piedra. Este pueblo gentil, pues, cuando lance una persecución contra el pueblo cristiano, será castigado con esta pena, será sumergido con su sacrílega piedra de molino en los flujos del siglo, que debe ser juzgado.

Traductores y estudiosos de la obra maximiana han asumido que el autor refería en este pasaje a un único colectivo al que denominaba indistintamente gentilis y paganus. Boniface Ramsey (1989:114), por ejemplo, en tanto que tradujo paganorum por "of the pagans", empleó tres veces 'gentil' y una vez 'pagan' para dar cuenta de las cuatro alusiones de Máximo a los gentiles contenidas en este sermón. Idéntica es la situación en la traducción al italiano de Giovanni Banterle (2003:203-204). El breve análisis del sermón XLVIII realizado por Maria Pia Amelia Ariano contiene numerosas afirmaciones que evidencian la condición de sinónimos que también la autora adjudicó a ambos términos. Sirva como muestra el siguiente caso: "Ricorda [Máximo], inoltre, che nelle Scritture con la figura dell'asino viene indicato il populus gentilium. I pagani 
infatti, rileva Massimo, girano sempre intorno al loro error..."(2014:229). Lo mismo cabe decir en relación a Maria Luisa Ariano, quien señaló que Máximo "ammonisce il popolo pagano assicurando che, se perseguiterà il popolo cristiano, la pena che lo attende sarà quella di essere immerso con la sua macina sacrilega nei flutti del mondo che deve essere giudicato"(2014:149).

A nuestro entender, el hecho de que Máximo recurriese a dos términos distintos exige examinar inicialmente si, por el contrario, no aludía a dos entidades diferentes; en tal caso, será necesario aprehender luego la especificidad de uno y otro colectivo.

En relación a lo primero, creemos que hay suficientes argumentos para sostener la independencia semántica entre los términos. En primer lugar, merece destacarse que el texto que servía a Máximo de guía para elaborar su propio comentario sobre el pasaje evangélico - esto es, las líneas 739-767 del capítulo VIII de la Expositio Evangelii Secundum Lucam de Ambrosio de Milán - aludía claramente a dos colectivos diversos, gentiles y Iudaei. ${ }^{33} \mathrm{El}$ hecho de que Máximo decidiese reemplazar este último por los pagani debe entenderse como una variación en el marco de un modelo, el ambrosiano, compuesto por dos elementos claramente diferentes. ${ }^{34}$

Más aún $-\mathrm{y}$ he aquí un segundo argumento - la estructura del pasaje maximiano se basa en una contraposición entre dos molae: por un lado, la mola asinaria en torno a la cual eran arrastrados los gentiles; por otro, la paganorum mola. A decir de Máximo se trataba de dos piedras distintas, relativas a dos sujetos diversos, cuya oposición quedaba claramente señalada por la conjunción adversativa sed.

Finamente, resulta decisiva la caracterización que de pagani y gentiles realizó Máximo en este pasaje, no solo desigual sino incluso opuesta en algunos puntos fundamentales. Los gentiles eran tenidos por presos del error, de la ignorantia, de la caecitas, de la inperitia, del labor y del extravío. Pero su condición era padecida de modo pasivo e involuntario, tal como señalaba el obispo al decir que eran llevados por la fuerza (pertrahitur) en el círculo de su error. Más aún, el gentilis estaba naturalmente llamado (Naturae quidem uinculis alligatus) a encontrar la fe - "a moler la palabra de Dios y a buscar al Señor del cielo" - y, con ella, la salvación. El error y la caecitas del paganus, en tanto, eran consecuencia directa de su accionar: había rechazado (recusauit) portare iugum domini. Por ello, afirmaba Máximo, habría de ser arrojado a lo profundo del mar con una piedra de molino de asno atada al cuello, es decir, habría de merecer la muerte eterna. Para el gentilis que importunase a los cristianos, por el contrario, el castigo establecido no preveía un padecimiento en el plano escatológico, sino que habría de ser sumergido saeculi fluctibus, esto es, habría de ser sometido a una pena terrenal, acaso consistente en la perpetuación de su desgraciada situación.

33 Por los motivos señalados en la nota 31, remitimos a Noce (2019:185-195) para una presentación y una comparación detalladas de los comentarios realizados respetivamente por Ambrosio de Milán y Máximo de Turín respecto de Mateo 18, 6 .

34 El trabajo de referencia respecto de la influencia de Ambrosio de Milán en la homilética maximiana es Trisoglio (2001). Coincidimos con la aseveración del autor según la cual "Dai luoghi convergenti risulta che Massimo non rifiuta i suggerimenti che ritiene valevoli, li accetta, ma senza lasciarsene dominare; sa appropriarseli e camminare in proprio" (Trisoglio, 2001:633). Almut Mutzenbecher había llegado a conclusiones similares en Mutzenbecher (1961:214-216). 


\section{Conclusiones}

Estas breves consideraciones sobre las líneas 78-102 del sermón 48 de Máximo de Turín sugieren que, de modo contrario a cuanto se ha tradicionalmente sostenido, gentilitas y paganismus daban cuenta para el autor de dos entidades históricas diferentes entre sí. La gentilitas maximiana puede ser entendida a partir de esta homilía como un estado de naturaleza primigenio en el cual el ser humano se hallaba desde su nacimiento hasta el momento en el cual la fe se manifestaba en él. En atención a su inocencia, resulta comprensible que Máximo no emitiese respecto de los gentiles un juicio negativo ni en este sermón ni en ninguno de los muchos en que la temática gentil se hacía presente. Los pagani, por el contrario, sí merecieron una consideración negativa por parte del obispo: eran sujetos que de algún modo habían entrado en contacto con el cristianismo y lo habían rechazado, mereciendo por ello el castigo eterno. Desconocimiento de la fe, capacidad para su aceptación y para la salvación; conocimiento de la fe, rechazo y castigo: tales eran los rasgos distintivos que en este sermón atribuía el obispo respectivamente a la gentilitas y al paganismus.

A esto solo cabe añadir aquí un par de observaciones. En primer lugar, señalar que - de acuerdo a nuestras indagaciones actualmente en curso - tanto la independencia semántica de los vocablos como la caracterización de la gentilitas aquí señalados no son exclusivos del sermón 48 sino que subyacen al conjunto de los Sermones. En efecto, la caracterización de la gentilitas como un estado natural pasible de ser superado reaparece en los sermones 20, 33, 35 y 84, en tanto que unos u otros de los rasgos atribuidos a los gentiles en estas homilías se reiteran en los restantes 17 sermones en que la cuestión gentil se hace presente ${ }^{35}$. Igual de importante, en ninguno de los textos maximianos relativos a ella se asocia a la gentilidad con los rasgos distintivos del paganismus, esto es, ni con el rechazo del cristianismo ni con el castigo eterno. Ambas circunstancias dan cuenta pues, de una conceptualización de la gentilitas no solo homogénea sino independiente del paganismus a lo largo de toda la obra homilética maximiana.

Por otra parte, cabe señalar que, lejos de reducirse a un mero análisis terminológico, nuestra propuesta pretende contribuir al debate respecto de la situación en que el proceso de cristianización se hallaba para tiempos de Máximo en Turín en particular y en el Piamonte en general. La determinación de la distinción maximiana entre gentilitas y paganismus, de sus características específicas y de la atención marcadamente mayor que la primera ameritaba para el obispo en comparación con la segunda, nos obligan a reconsiderar el escenario tradicionalmente planteado por la historiografía, de acuerdo al cual el cristianismo local debía hacer frente a un enemigo tenaz, el 'paganismo', que habría constituido una poderosa alternativa a su desarrollo. En efecto, si la prédica del obispo insistía reiteradamente en la necesidad de invitar a la fe a aquellos individuos que la ignoraban - los gentiles - y de instruir a los propios cristianos procedentes de la gentilitas respecto de las exigencias de la

35 Valgan algunos ejemplos. Alusiones a la potencial redención de los gentiles en S. II, 54-56; S. XX, 52-69; S. XXXIII, 82-85; S. XXXV, 82-89; S. XLVIII, 93-94; S. LXIc extr., 65-72; S. LXXIII, 46-50; S. LXXXIV, 87-93; S. XCI extr., 37-39; S. XCVIII extr., 11-20; S. CIII, 20-29; S. CV extr., 49-51; S. CVI extr., 20-21. Asociaciones de los gentiles con el error. S. XXX, 52-53; S. XXXI, 6; S. XLVIII, 80; S. LXIc extr., 65-66; S. XCVIII extr., 4; S. CV extr., 35; S. CVI extr., 25; S. CVII extr., 5-6; asociaciones de los gentiles con el sacrilegium: S. XXX, 45-47; S. XLVIII, 99-102; S. LXXXIV, 88-89; S. LXXXV, 58-59; S. XCVIII extr., 5-7; S. CV extr., 33, 44-45; S. CVI extr., 4, 8, 14, 24, 29, 38, 45; S. CVII extr., 10-11, 25-26; asociaciones de los gentiles con la superstitio: S. I, 36; S. LXIc extr., 72; S. LXIII, 5; S. LXXIII, 95-102; S. XCVIII extr., 4, 5-6, 17-19; S. Cl extr., 7-11; S. CIII extr., 28; S. CVI extr., 5. Para un examen exhaustivo de las características asignadas por Máximo a los gentiles y su reiteración en el conjunto de los sermones en que la gentilitas es evocada, véase Noce (2019: en prensa).. 
fe abrazada, acaso debamos asumir que no preocupaba tanto a Máximo una amenaza semejante - carente de reflejo en sus sermones - sino las dificultades que el cristianismo encontraba para difundirse y asentarse en el seno de una población que, lejos de oponerse a él, el autor aguardaba se mostrase proclive a aceptarlo una vez que lo conociese. En otras palabras, creemos que la pregunta acerca de cómo combatir una amenaza para la expansión del cristianismo no inquietó tanto a Máximo como aquella respecto de por qué vías la nueva fe podía expandir su todavía acotada presencia e influencia. ${ }^{36}$

36 Los testimonios arqueológicos y epigráficos refrendan el carácter aún incipiente del proceso de cristianización. Con relación a Turín en particular y al Piamonte en general, Cantino Wataghin (1997 y 1999). Para una mirada de más amplio alcance, Guyon; Baratte; Cantino Wataghin; Heijmans (2016), estudio en el cual, a partir de un exhaustivo repaso de los testimonios literarios, arqueológicos y epigráficos relativos a la presencia cristiana tanto en el ámbito urbano como en el rural, se señala la conveniencia de retrasar hasta después del año 380 "le parachèvement du «tournant constantinien»" (2016:37). 


\section{Q Bibliografía}

» Ariano, M.L. (2014). “La condanna dell'idolatria nell'omiletica dell'Italia settentrionale di fine IV secolo", Auctores Nostri 14, 129-163.

》 Ariano, M.L. (2017). "Polemica per immagini aduersus paganosnell'omiletica dell'Italia settentrionale di fine IV secolo", Auctores Nostri 18, 11-35.

"Ariano, M.P.A. (2014). "La polemica antipagana in Massimo di Torino", Auctores Nostri $14,211-242$.

» Banterle, G. (ed.) (1991). San Massimo di Torino. Sermoni. Milano-Roma: Città Nuova.

» Banterle, G. (trad.); Petri S. (intr. y notas) (2003). Massimo di Torino. Sermones. Roma: Città Nuova.

" Bolgiani, F. (1997). "Massimo di Torino, la sua personalità, la sua predicazione, il suo pubblico". En: Sergi, G. (ed.), Storia di Torino. I. Dalla preistoria al comune medievale. Torino: Einaudi, 255-269.

" Bolgiani, F. (1998). "La diocesi di Torino nel IV-V secolo sotto i due Massimo". En: Mercando, L.; Micheletto, E. (eds.), Archeologia in Piemonte. Volume III. Il Medioevo. Torino: Allemandi, 121-131.

"Cantino Wataghin, G. (1997). "La cristianizzazione dello spazio urbano". En: Sergi, G. (ed.), Storia di Torino. I. Dalla preistoria al comune medievale. Torino: Einaudi, 255269.

" Cantino Wataghin, G. (1998) "Dinamiche della cristianizzazione nella diocesi di Torino: le testimonianze archeologiche". En: Atti del Convegno Internazionale di Studi su Massimo di Torino nel XVI Centenario del Concilio di Torino (398) (Torino, 13-14 marzo 1998). Torino: Elledici, 18-49.

" De Filippis Cappai, Ch. (1995). Massimo Vescovo di Torino e il suo tempo. Torino: Società Editrice Internazionale.

"De Filippis Cappai, Ch. (1999). "Società e cristianesimo a Torino al tempo di Massimo". En: Atti del Convegno Internazionale di Studi su Massimo di Torino nel XVI Centenario del Concilio di Torino (398) (Torino, 13-14 marzo 1998). Torino: Elledici, 50-68 .

"Devoti, D. (1981). "Massimo di Torino e il suo pubblico", Augustinianum 21, 1, 143167.

"Devoti, D. (1999). "Massimo oratore". En: Atti del Convegno Internazionale di Studi su Massimo di Torino nel XVI Centenario del Concilio di Torino (398) (Torino, 13-14 marzo 1998). Torino: Elledici, 99-115.

"Di Mauro, N. (2001). La paterna tenerezza di un pastore d'anime. San Massimo vescovo di Torino. Torino: Effatà.

"Eger, G. (2014). Magna Pollutio: Saint Maximus on the Survival of Paganism in 4th century Turin. A senior thesis submitted to the Classics Department of Princeton University in partial fulfillment of the requirements for the degree of Bachelor of Arts. Princeton. https://dataspace.princeton.edu/ispui/handle/88435/dsp0141687h63t.

" Gallesio, F. (ed.) (1975). S. Massimo di Torino, Sermoni. Alba: Paoline.

"Guyon, J.; Baratte, F.; Cantino Wataghin, G.; Heijmans, M. (2016). "La diffusion du christianisme et ses incidences topographiques sur les villes et les campagnes de l'Occident constantinien". En: Brandt, O., Fiocchi Nicolai, V., Castiglia, G. (eds.), 
Costantino e i costantinidi. L'innovazione costantiniana, le sue radici e i suoi sviluppi (Acta XVI Congressus Internationalis Archaeologiae Christianae. Romae (2228.9.2013). Città del Vaticano: Pontificio Istituto di Archeologia Cristiana, 3-109.

» Lizzi, R. (1989). Vescovi e strutture ecclesiastiche nella città tardoantica (l'Italia Annonaria nel IV-V secolo d.C.). Como: New Press.

» Mariani Puerari, M. (ed.) (1999). Massimo di Torino. Sermoni Liturgici. Milano: Paoline.

» Maritano, M. (1999). "La sacra scrittura nei Sermoni e nel ministero episcopale di Massimo di Torino". En Atti del Convegno Internazionale di Studi su Massimo di Torino nel XVI Centenario del Concilio di Torino (398) (Torino, 13-14 marzo 1998). Torino: Elledici, 116-166.

" Merkt, A. (1997). Maximus I. von Turin: die Verkundigung eines Bischofs der fruhen Reichskirche im zeitgeschichtlichen, gesellschaftlichen und liturgischen Kontext. Leiden-New York-Köln: Brill.

" Mutzenbecher, A. (1961). "Bestimmung der echten Sermones des Maximus Taurinensis", SEJG 12, 197-293.

» Mutzenbecher, A. (ed.) (1962). Maximi episcopi Taurinensis sermones (CCSL, XXIII). Turnhout: Brepols.

" Noce, E. (2017). "Cristianismo y gentilitas en los Sermones de Máximo de Turín: consideraciones críticas sobre el estado de la cuestión", VetChr 54, 173-204.

» Noce, E. (2019). "Gentilitas y paganismus en los sermones de Máximo de Turín: reconsideración de una sinonimia infundada", AnnSE 36, 1, 175-203.

"Noce, E. (en prensa). "En torno a la independencia semántica de los términos gentilitas y paganismus en los Sermones de Máximo de Turín. Verificación de una hipótesis", AntTard, 27.

" Padovese, L. (ed.) (1985). Sermoni di S. Massimo di Torino (2 volúmenes). Casale Monferrato: Piemme.

»Padovese, L. (1999). "Massimo vescovo di Torino". En Atti del Convegno Internazionale di Studi su Massimo di Torino nel XVI Centenario del Concilio di Torino (398) (Torino, 13-14 marzo 1998). Torino: Elledici, 85-98.

» Plazanet-Siarri, N. (trad.); Hamman, A.-G. (intr.) (1996). Maxime de Turin prêche l'année liturgique. Paris: Brepols.

» Ramsey, B. (ed.) (1989). The Sermons of St. Maximus of Turin. New York-Mahwah: Newman Press.

» Spinelli, L. (1983). Maria nei Sermoni di S. Massimo vescovo di Torino. Vicenza: Edizioni Patristiche.

"Trisoglio, F. (2001). "S. Ambrogio e l'esegesi di S. Massimo di Torino", AnnSE 18, 2, 615-635.

"Zangara, V. (1992). "I 'mandata' divini nella predicazione di Massimo di Torino", AnnSE 9, 2, 493-518. 